\title{
Time damping of non-adiabatic magnetohydrodynamic waves in a partially ionized prominence plasma: effect of helium
}

\author{
R. Soler, R. Oliver, and J. L. Ballester \\ Departament de Física, Universitat de les Illes Balears, 07122 Palma de Mallorca, Spain \\ e-mail: [roberto.soler;ramon.oliver; joseluis.ballester]@uib.es \\ Received 15 October 2009 / Accepted 14 December 2009

\section{ABSTRACT}

\begin{abstract}
Context. Prominences are partially ionized, magnetized plasmas embedded in the solar corona. Damped oscillations and propagating waves are commonly observed. These oscillations have been interpreted in terms of magnetohydrodynamic (MHD) waves. Ion-neutral collisions and non-adiabatic effects (radiation losses and thermal conduction) have been proposed as damping mechanisms.

Aims. We study the effect of the presence of helium on the time damping of non-adiabatic MHD waves in a plasma composed by electrons, protons, neutral hydrogen, neutral helium (He I), and singly ionized helium (He II) in the single-fluid approximation.

Methods. The dispersion relation of linear non-adiabatic MHD waves in a homogeneous, unbounded, and partially ionized prominence medium is derived. We compute the period and the damping time of Alfvén, slow, fast, and thermal waves. A parametric study of the ratio of the damping time to the period with respect to the helium abundance is performed.

Results. The efficiency of ion-neutral collisions, as well as thermal conduction, is increased by the presence of helium. However, if realistic abundances of helium in prominences $(\sim 10 \%)$ are considered, this has a minor influence on the wave damping.

Conclusions. The presence of helium can be safely neglected in studies of MHD waves in partially ionized prominence plasmas.
\end{abstract}

Key words. Sun: oscillations - magnetic fields - Sun: corona - Sun filaments, prominences

\section{Introduction}

Small-amplitude oscillations and propagating waves are commonly observed in both quiescent and active region prominences/filaments. They have been interpreted in terms of magnetohydrodynamic (MHD) eigenmodes of the magnetic structure and/or propagating MHD waves. The reader is referred to some recent reviews for more information about the observational and theoretical backgrounds (Oliver \& Ballester 2002; Engvold 2004; Ballester 2006; Banerjee et al. 2007; Engvold 2008).

Prominence oscillations are known to be quickly damped, with damping times corresponding to a few oscillatory periods (reviewed by Oliver 2009; Mackay et al. 2010). Several damping mechanisms of MHD waves have been proposed, with nonadiabatic effects and ion-neutral collisions the more extensively investigated. To understand these effects in detail, they have been studied in simple configurations such as unbounded and homogeneous media. Carbonell et al. (2004) investigated the time damping in a homogeneous prominence medium taking nonadiabatic effects (optically thin radiation losses and thermal conduction) into account. Later on, the spatial damping was studied by Carbonell et al. (2006) and the effect of a background mass flow was analyzed by Carbonell et al. (2009). Subsequently, some works have extended these previous results by considering the presence of the coronal medium (Soler et al. 2007, 2008, 2009a). The common conclusion of these investigations is that only slow and thermal waves are efficiently damped by nonadiabatic effects, while fast waves are very slightly damped and Alfvén waves are completely unaffected.

On the other hand, the influence of partial ionization on the propagation and time damping of MHD waves has been also investigated in an unbounded medium. Forteza et al. (2007) follow the treatment by Braginskii (1965) to derive the full set of MHD equations along with the dispersion relation of linear waves in a partially ionized, single-fluid plasma (see also Pinto et al. 2008). The electrons, protons, and neutral hydrogen atoms were taken into account, whereas helium and other species were not considered. In a subsequent work (Forteza et al. 2008), they extended their previous analysis by considering radiative losses and thermal conduction by electrons and neutrals. Their main results with respect to the fully ionized case (Carbonell et al. 2004) were, first of all, that ion-neutral collisions (by means of the so-called Cowling's diffusion) can damp both Alfvén and fast waves but non-adiabatic effects remain only important for the damping of slow and thermal waves, and second, that critical values of the wavenumber exist in which the real part of the frequency vanishes, so wave propagation is impossible for larger wavenumbers. Again, applications to a more complex cylindrical geometry have also been performed (Soler et al. 2009b,c).

On the basis of these previous results, it seems clear that partial ionization plays a relevant role on wave propagation in prominences. Prominences are roughly composed of $90 \%$ hydrogen and $10 \%$ helium but, to date, all the investigations considered a pure hydrogen plasma. Therefore, the effect of the helium on the propagation and damping of MHD waves is still unknown and is the motivation for the present work. Here, we consider an unbounded and homogeneous prominence medium permeated by a homogeneous magnetic field. The plasma is assumed to be partially ionized, because electrons, protons, neutral hydrogen, neutral helium (He I), and singly ionized helium (He II) are the species taken into account. Recent studies by Gouttebroze \& Labrosse (2009) indicate that, for central prominence temperatures, the ratio of the number densities of $\mathrm{He}$ II to He I is 
around $10 \%$, whereas the presence of He III is negligible. This result allows us to neglect $\mathrm{He}$ III in this work. Extending the works by Forteza et al. $(2007,2008)$, the derivation of the basic MHD equations for a non-adiabatic, partially ionized, singlefluid plasma has been generalized by now considering five different species, allowing us to study how the presence of neutral and singly ionized helium affects their previous results.

This paper is organized as follows. The description of the equilibrium and the basic equations are given in Sect. 2. The results are discussed in Sect. 3. Finally, Sect. 4 contains the conclusion of this work.

\section{Equilibrium and basic equations}

Our equilibrium configuration is a homogeneous and unbounded partially ionized plasma composed by electrons, protons, neutral hydrogen, neutral helium, and singly ionized helium. Hereafter, subscripts e, p, H, He I, and He II explicitly denote these species, respectively. The magnetic field is also homogeneous and orientated along the $x$-direction, $\boldsymbol{B}=B_{0} \hat{e}_{x}$, with $B_{0}$ constant. We adopt the single-fluid approximation. Following Forteza et al. (2007, 2008) and neglecting the electron contribution, we define the center-of-mass velocity, $\boldsymbol{v}$, as

$\boldsymbol{v} \approx \xi_{\mathrm{p}} \boldsymbol{v}_{\mathrm{p}}+\xi_{\mathrm{H}} \boldsymbol{v}_{\mathrm{H}}+\xi_{\mathrm{He} \text { I }} \boldsymbol{v}_{\mathrm{He}}+\xi_{\mathrm{He} \mathrm{II}} \boldsymbol{v}_{\mathrm{He} \text { II }}$,

with $\xi_{\alpha}$ the relative density of species $\alpha$, and $\boldsymbol{v}_{\alpha}$ the corresponding species velocity. Equivalently, the equilibrium total density, $\rho$, and gas pressure, $p$, are

$\rho \approx \rho_{\mathrm{p}}+\rho_{\mathrm{H}}+\rho_{\mathrm{He}}+\rho_{\mathrm{He} I \mathrm{II}}$,

$p=2\left(p_{\mathrm{p}}+p_{\mathrm{He} \text { II }}\right)+p_{\mathrm{H}}+p_{\text {He I }}$.

Since $\rho_{\alpha}=\xi_{\alpha} \rho$, we get the relation $\xi_{\mathrm{p}}+\xi_{\mathrm{H}}+\xi_{\mathrm{He} \text { I }}+\xi_{\mathrm{He} \mathrm{II}} \approx 1$. We assume a strong thermal coupling between species, so all the species have the same equilibrium temperature $T$. Then, the three equilibrium quantities are related as

$p=\rho \frac{R}{\tilde{\mu}} T$,

where $R$ is the ideal gas constant and $\tilde{\mu}$ the mean atomic weight,

$\tilde{\mu}=\frac{1}{2 \xi_{\mathrm{p}}+\xi_{\mathrm{H}}+\frac{1}{4} \xi_{\mathrm{He} \text { I }}+\frac{1}{2} \xi_{\mathrm{He} \text { II }}}$.

We can express $\tilde{\mu}$ in a more convenient form,

$\tilde{\mu}=\frac{\tilde{\mu}_{\mathrm{H}}}{1-\left[\left(1+\delta_{\mathrm{He}}\right)+\left(1+2 \delta_{\mathrm{He}}\right) \frac{1}{4} \tilde{\mu}_{\mathrm{H}}\right] \xi_{\mathrm{He}}}$,

here we have defined

$\tilde{\mu}_{\mathrm{H}}=\frac{\xi_{\mathrm{p}}+\xi_{\mathrm{H}}}{2 \xi_{\mathrm{p}}+\xi_{\mathrm{H}}}, \quad \delta_{\mathrm{He}}=\frac{\xi_{\mathrm{He} \text { II }}}{\xi_{\mathrm{He}}}$.

The quantity $\tilde{\mu}_{\mathrm{H}}$ is equivalent to the mean atomic weight of a pure hydrogen plasma defined in Eq. (3) of Forteza et al. (2007), and ranges between $\tilde{\mu}_{\mathrm{H}}=0.5$ for a fully ionized hydrogen plasma and $\tilde{\mu}_{\mathrm{H}}=1$ for a fully neutral hydrogen gas. On the other hand, $\delta_{\mathrm{He}}$ indicates the helium ionization degree. A realistic value of this parameter is $\delta_{\mathrm{He}}=0.1$ according to the results of Gouttebroze \& Labrosse (2009). From Eq. (6) one can see that $\tilde{\mu}>\tilde{\mu}_{\mathrm{H}}$ because of the presence of helium. In the absence of helium, $\xi_{\mathrm{He}}=\xi_{\mathrm{He} \text { II }}=0$ so $\tilde{\mu}=\tilde{\mu}_{\mathrm{H}}$. Figure 1 displays the dependence of $\tilde{\mu}$ on $\xi_{\mathrm{He}}$ for several values of $\tilde{\mu}_{\mathrm{H}}$.

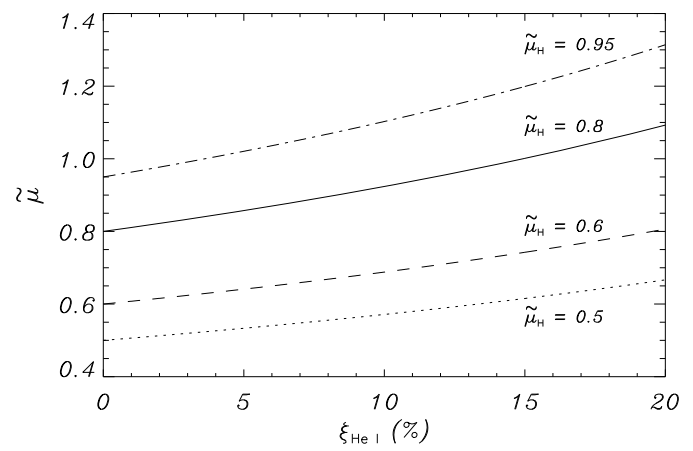

Fig. 1. Mean atomic weight, $\tilde{\mu}$, as a function of the relative neutral helium density, $\xi_{\mathrm{He}}$, for $\tilde{\mu}_{\mathrm{H}}=0.5$ (dotted line), $\tilde{\mu}_{\mathrm{H}}=0.6$ (dashed line), $\tilde{\mu}_{\mathrm{H}}=0.8$ (solid line), and $\tilde{\mu}_{\mathrm{H}}=0.95$ (dash-dotted line). In all cases, $\delta_{\mathrm{He}}=0.1$.

The details of the derivation of the basic governing equations for a non-adiabatic, partially ionized, one-fluid plasma can be followed in, e.g., Braginskii (1965), Forteza et al. (2007, 2008), Pinto et al. (2008). Here, we follow the same procedure but generalize the analysis of Forteza et al. (2007) by including additional species. In brief, the separate governing equations for the five species are added and a generalized Ohm's law is obtained. A key step in the present derivation is to compute the density current, $j$, as

$\boldsymbol{j}=e\left(n_{\mathrm{p}} \boldsymbol{v}_{\mathrm{p}}+n_{\mathrm{He} \text { II }} \boldsymbol{v}_{\mathrm{He} \text { II }}-n_{\mathrm{e}} \boldsymbol{v}_{\mathrm{e}}\right)$,

along with the condition $n_{\mathrm{e}}=n_{\mathrm{p}}+n_{\mathrm{He} \text { II }}$, where $n_{\mathrm{e}}, n_{\mathrm{p}}$, and $n_{\mathrm{He} I \mathrm{I}}$ are the electron, proton, and He II number densities, respectively, and $e$ the electron charge. The resulting general induction equation (see Eq. (14) of Forteza et al. 2007) contains several diffusion terms whose coefficients depend on the collisional frequencies between species. The physical meaning of these nonideal terms is explained in detail in Pinto et al. (2008). In particular, ion-neutral collisions are responsible for the so-called Cowling's diffusion, which is much more efficient than Ohm's diffusion in a partially ionized plasma. However, some terms are not relevant for our present application. Hall's effect is negligible in prominence conditions (Soler et al. 2009c), and the so-called "Biermann's battery" term is identically zero in a homogeneous medium. For this reason, our final form of the induction equation only contains the terms corresponding to Ohm's and ambipolar (Cowling's) diffusion, along with the diamagnetic current term. Therefore, our basic equations are

$\frac{\mathrm{D} \rho}{\mathrm{D} t}+\rho \nabla \cdot \boldsymbol{v}=0$

$\rho \frac{\mathrm{D} v}{\mathrm{D} t}=-\nabla p+\frac{1}{\mu}(\nabla \times \boldsymbol{B}) \times \boldsymbol{B}$,

$\frac{\mathrm{D} p}{\mathrm{D} t}-\frac{\gamma p}{\rho} \frac{\mathrm{D} \rho}{\mathrm{D} t}+(\gamma-1)[\rho L(T, \rho)-\nabla \cdot(\kappa \cdot \nabla T)]=0$,

$$
\begin{gathered}
\frac{\partial \boldsymbol{B}}{\partial t}=\nabla \times(\boldsymbol{v} \times \boldsymbol{B})+\eta \nabla^{2} \boldsymbol{B}-\Xi \nabla \times[\nabla p \times \boldsymbol{B}] \\
+\frac{\eta_{\mathrm{C}}-\eta}{B_{0}^{2}} \nabla \times\{[(\nabla \times \boldsymbol{B}) \times \boldsymbol{B}] \times \boldsymbol{B}\},
\end{gathered}
$$

$\nabla \cdot \boldsymbol{B}=0$

$p=\frac{\rho R T}{\tilde{\mu}}$, 
where $\frac{\mathrm{D}}{\mathrm{D} t} \equiv \frac{\partial}{\partial t}+\boldsymbol{v} \cdot \nabla$ is the material derivative for time variations following the plasma motion, $\mu=4 \pi \times 10^{-7} \mathrm{~N} \mathrm{~A}^{-2}$ is the magnetic permeability, $\gamma=5 / 3$ is the adiabatic index, $\eta$ and $\eta_{\mathrm{C}}$ are the Ohm's and Cowling's coefficients of magnetic diffusion, respectively, $\Xi$ is the diamagnetic current coefficient, $L(T, \rho)$ is the heat-loss function, and $\kappa$ is the thermal conductivity. The quantities $\eta$ and $\eta_{\mathrm{C}}$ can be expressed in terms of their corresponding magnetic conductivities,

$\eta=\frac{1}{\mu \sigma}, \quad \eta_{\mathrm{C}}=\frac{1}{\mu \sigma_{\mathrm{C}}}$.

Thus, Ohm's and Cowling's conductivities, as well as the diamagnetic current coefficient, $\Xi$, which applies in our case when helium is included, are

$\sigma=\frac{e^{2} n_{\mathrm{e}}^{2}}{\left(\alpha_{\mathrm{e}}-\alpha_{\mathrm{en}}^{2} / \alpha_{\mathrm{n}}\right)}, \quad \sigma_{\mathrm{C}}=\frac{\sigma}{1+\frac{B_{0}^{2}\left(\xi_{\mathrm{H}}+\xi_{\mathrm{He}}\right)^{2}}{\alpha_{\mathrm{n}}} \sigma}$

$\Xi=\frac{\left(\xi_{\mathrm{H}}+\xi_{\mathrm{He}}\right)}{\tilde{\mu} \alpha_{\mathrm{n}}}\left(\xi_{\mathrm{p}} \xi_{\mathrm{H}}-\frac{1}{2} \xi_{\mathrm{H}} \xi_{\mathrm{He} \mathrm{II}}+\frac{7}{4} \xi_{\mathrm{p}} \xi_{\mathrm{He} \text { I }}+\frac{1}{4} \xi_{\mathrm{He} \mathrm{I}} \xi_{\mathrm{He} \mathrm{II}}\right)$

In addition, $\alpha_{\mathrm{e}}, \alpha_{\mathrm{en}}$, and $\alpha_{\mathrm{n}}$ are the electron, electron-neutral, and neutral friction coefficients, respectively, whose expressions depend on the sum of the friction coefficients between particular species,

$\alpha_{\mathrm{en}}=\alpha_{\mathrm{eH}}+\alpha_{\mathrm{eHe}}$,

$\alpha_{\mathrm{e}}=\alpha_{\mathrm{ep}}+\alpha_{\mathrm{eH}}+\alpha_{\mathrm{eHe} \text { I }}+\alpha_{\mathrm{eHe} \text { II }}$

$\alpha_{\mathrm{n}}=\alpha_{\mathrm{eH}}+\alpha_{\mathrm{eHe}}+\alpha_{\mathrm{pH}}+\alpha_{\mathrm{pHeI}}+\alpha_{\mathrm{He} \text { IIH }}+\alpha_{\mathrm{He} \text { IIHe I }}$.

Each particular friction coefficient, $\alpha_{\beta \beta^{\prime}}$, is computed as

$\alpha_{\beta \beta^{\prime}}=n_{\beta} m_{\beta \beta^{\prime}} v_{\beta \beta^{\prime}}$,

with $n_{\beta}$ the number density of the species $\beta, v_{\beta \beta^{\prime}}$ the collisional frequency between species $\beta$ and $\beta^{\prime}$, and

$m_{\beta \beta^{\prime}}=\frac{m_{\beta} m_{\beta^{\prime}}}{m_{\beta}+m_{\beta^{\prime}}}$,

with $m_{\beta}$ the mass particle of the species $\beta$. As given by De Pontieu et al. (2001), see also Soler et al. (2009b), the collisional frequencies between electrons and protons or He II are

$v_{\mathrm{ei}}=3.7 \times 10^{-6} \frac{n_{\mathrm{i}} \ln \Lambda}{T_{0}^{3 / 2}}$,

with $\mathrm{i}=\mathrm{p}$ or He II and $\ln \Lambda$ the Coulomb logarithm, while the collisional frequency between a charged species, $q=e, p$, or $\mathrm{He}$ II, and a neutral species, $\mathrm{n}=\mathrm{H}$ or $\mathrm{He} \mathrm{I}$, is

$v_{\mathrm{qn}}=n_{\mathrm{n}} \sqrt{\frac{8 k_{\mathrm{B}} T_{0}}{\pi m_{\mathrm{qn}}}} \Sigma_{\mathrm{qn}}$,

with $k_{\mathrm{B}}$ the Boltzmann's constant, and $\Sigma_{\mathrm{qn}}$ the collisional crosssection. Here, we consider the values $\Sigma_{\mathrm{en}}=10^{-19} \mathrm{~m}^{2}$, and $\Sigma_{\mathrm{pn}}=$ $\Sigma_{\text {He IIn }}=5 \times 10^{-19} \mathrm{~m}^{2}$.

On the other hand, the thermal conductivity due to neutrals (Eq. (16) of Forteza et al. 2008) now has to include the helium contribution. According to Parker (1953), a corrected expression for the conductivity of neutrals in MKS units is

$\kappa_{\mathrm{n}}=\kappa_{\mathrm{H}}+\kappa_{\mathrm{He}}=\left(2.44 \times 10^{-2} \xi_{\mathrm{H}}+3.18 \times 10^{-2} \xi_{\mathrm{He}}\right) T^{1 / 2}$.
Finally, we assume an optically thin radiation (Hildner 1974) to represent the hydrogen radiative losses. According to Cox \& Tucker (1969, see their Fig. 3), the radiative losses by helium are several orders of magnitude less than those of hydrogen for typical prominence temperatures $\left(\sim 10^{4} \mathrm{~K}\right)$, hence irrelevant for the present investigation.

Hereafter, our analysis follows that of Forteza et al. (2008). We linearize the basic equations and assume small perturbations proportional to $\exp \left(\mathrm{i} \omega t+\mathrm{i} k_{x} x+\mathrm{i} k_{z} z\right)$. Then the resulting equations (Eq. (18)-(27) of Forteza et al. 2008) are combined and finally two different, uncoupled dispersion relations, one for Alfvén waves (their Eq. (28)) and another for magnetoacoustic and thermal waves (their Eq. (30)), are obtained. Although our definitions of $\eta, \eta_{\mathrm{C}}, \Xi$, and $\kappa_{\mathrm{n}}$ contain the effect of helium, the resulting dispersion relations are formally identical to those of Forteza et al. (2008). For the sake of simplicity, we do not write these expressions here again and refer the reader to that paper. The dispersion relations are numerically solved for real values of the wavenumber modulus, $k=\sqrt{k_{x}^{2}+k_{z}^{2}}$, and the angle $\theta$ between $\boldsymbol{B}_{0}$ and $\boldsymbol{k}$. A complex frequency, $\omega=\omega_{\mathrm{R}}+\mathrm{i} \omega_{\mathrm{I}}$, is obtained. The period, $P$, and damping time, $\tau_{\mathrm{D}}$, are related to the real and imaginary parts of the frequency as

$P=\frac{2 \pi}{\omega_{\mathrm{R}}}, \quad \tau_{\mathrm{D}}=\frac{1}{\omega_{\mathrm{I}}}$

\section{Results}

In the following computations, unless otherwise stated, we consider typical quiescent prominence conditions, $\rho=5 \times$ $10^{-11} \mathrm{~kg} \mathrm{~m}^{-3}, T=8000 \mathrm{~K}$, and $B_{0}=5 \mathrm{G}$. Quantities $\tilde{\mu}_{\mathrm{H}}, \xi_{\mathrm{He}}$, and $\delta_{\mathrm{He}}$ are considered free parameters. We focus our attention on the effect of the relative neutral helium density, $\xi_{\mathrm{He} \text { I }}$, on the ratio $\tau_{\mathrm{D}} / P$.

\subsection{Free propagation in an unbounded medium}

First, we assume $\theta=\pi / 4$. Figure 2 displays $\tau_{\mathrm{D}} / P$ as a function of $k$ for the Alfvén, fast, and slow waves. The results corresponding to several helium abundances are compared for hydrogen and helium ionization degrees of $\tilde{\mu}_{\mathrm{H}}=0.8$ and $\delta_{\mathrm{He}}=0.1$, respectively. We see that even in the case of the largest quantity of helium considered $\left(\xi_{\mathrm{He}}=20 \%\right)$, the presence of helium has a minor effect on the results. In the case of Alfvén and fast waves (Fig. 2a,b), their critical wavenumber (i.e., the value of $k$ which causes the real part of the frequency to vanish) is shifted toward slightly lower values, so the larger $\xi_{\mathrm{He}}$, the smaller $k_{\mathrm{c}}^{\mathrm{a}}$. This result can be understood by considering that the Alfvén wave critical wavenumber, $k_{\mathrm{c}}^{\mathrm{a}}$, given by Eq. (38) of Forteza et al. (2008) is

$k_{\mathrm{c}}^{\mathrm{a}}=\frac{2 v_{\mathrm{A}}}{\left(\eta_{\mathrm{C}}+\eta \tan ^{2} \theta\right) \cos \theta}$,

with $v_{\mathrm{A}}=B_{0} / \sqrt{\mu \rho}$ the Alfvén speed. Equation (27) is also approximately valid for the fast wave critical wavenumber. Then, we see that $k_{\mathrm{c}}^{\mathrm{a}}$ is inversely proportional to Cowling's diffusivity, $\eta_{\mathrm{C}}$. Since $\eta_{\mathrm{C}}$ is larger in the presence of helium than in the pure hydrogen case, because of additional collisions of neutral and singly ionized helium species, $k_{\mathrm{c}}^{\mathrm{a}}$ is therefore smaller. Turning our attention to the slow wave (Fig. 2c), we see that the maximum and the right hand side minimum of $\tau_{\mathrm{D}} / P$ are also slightly shifted toward lower values of $k$. Results from Carbonell et al. (2004) and Forteza et al. (2008) indicate that thermal conduction is responsible for the maximum and minimum of $\tau_{\mathrm{D}} / P$. Thus, 

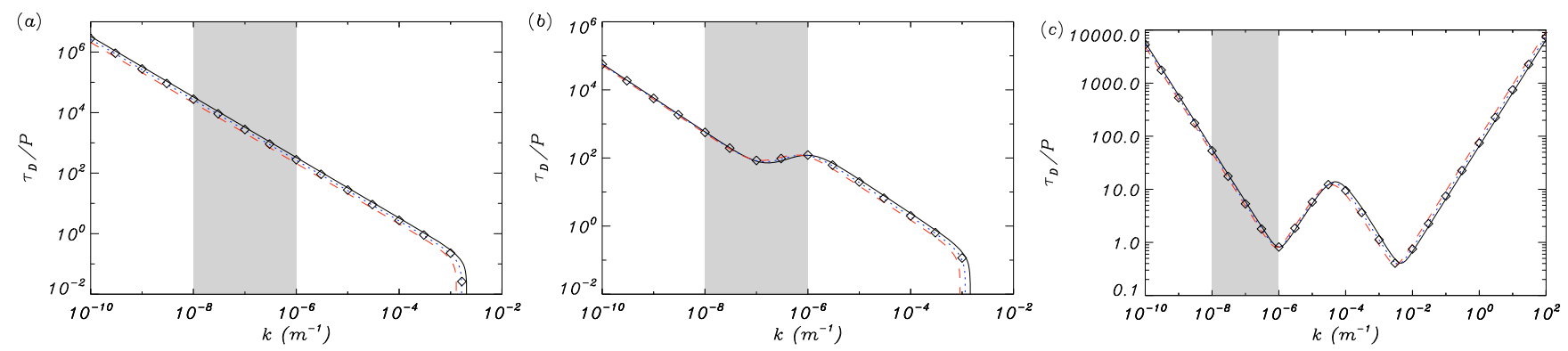

Fig. 2. Ratio of the damping time to the period, $\tau_{\mathrm{D}} / P$, versus the wavenumber, $k$, corresponding to the a) Alfvén wave, b) fast wave, and c) slow wave for $\theta=\pi / 4, \tilde{\mu}_{\mathrm{H}}=0.8$, and $\delta_{\mathrm{He}}=0.1$. The different linestyles represent $\xi_{\mathrm{HeI}}=0 \%$ (solid line), $\xi_{\mathrm{HeI}}=10 \%$ (dotted line), and $\xi_{\mathrm{HeI}}=20 \%$ (dashed line). The results for $\xi_{\mathrm{He}}=10 \%$ and $\delta_{\mathrm{He}}=0.5$ are plotted by means of symbols for comparison. The shaded regions correspond to the range of typically observed wavelengths of prominence oscillations.
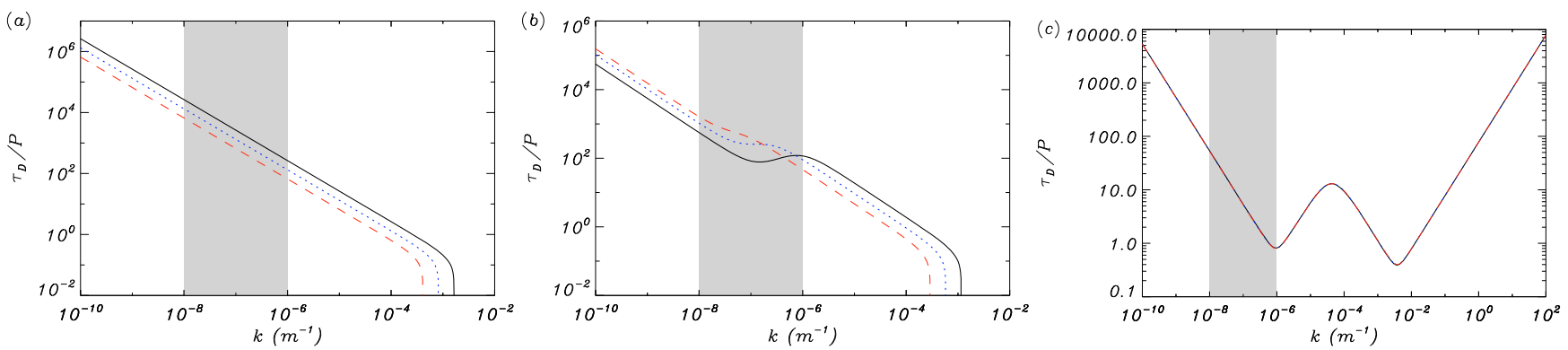

Fig. 3. Ratio of the damping time to the period, $\tau_{\mathrm{D}} / P$, versus the wavenumber, $k$, corresponding to the a) Alfvén wave, b) fast wave, and c) slow wave for $\theta=\pi / 4, \tilde{\mu}_{\mathrm{H}}=0.8, \xi_{\mathrm{He}}=10 \%$, and $\delta_{\mathrm{He}}=0.1$. The different linestyles represent $B_{0}=5 \mathrm{G}$ (solid line), $B_{0}=10 \mathrm{G}$ (dotted line), and $B_{0}=20 \mathrm{G}$ (dashed line).

the additional contribution of neutral helium atoms to thermal conduction (Eq. (25)) causes this displacement of the curve of $\tau_{\mathrm{D}} / P$. As for Alfvén and fast waves, this effect is not very important. For comparison, equivalent results with $\xi_{\mathrm{He}}=10 \%$ and $\delta_{\mathrm{He}}=0.5$ are plotted by means of symbols in Fig. 2 . We see that, for realistic values of $\delta_{\mathrm{He}}$, its role is almost irrelevant, meaning that the presence of He II can be neglected. It is worth mentioning that we repeated these calculations for other values of $\tilde{\mu}_{\mathrm{H}}$ and had similar results.

Now, Fig. 3 shows the dependence of $\tau_{\mathrm{D}} / P$ on the magnetic field strength, $B_{0}$. The value of Cowling's diffusion, $\eta_{\mathrm{C}}$, is proportional to $B_{0}^{2}$ (see Eqs. (15) and (16)). For this reason, the Alfvén mode is more attenuated as $B_{0}$ grows, since Cowling's diffusion is more efficient. The fast wave shows a similar behavior for large $k$, whereas for small $k$, including the region on typical wavelengths, the behavior is the opposite because the damping is dominated by thermal mechanisms instead of ion-neutral collisions. As expected, the slow mode shows no dependence on the value of the magnetic field strength.

Next, we study the thermal mode. Since it is a purely damped, non-propagating disturbance $\left(\omega_{\mathrm{R}}=0\right)$, we only plot the damping time, $\tau_{\mathrm{D}}$, as a function of $k$ for $\tilde{\mu}_{\mathrm{H}}=0.8$ and $\delta_{\mathrm{He}}=0.1$ (Fig. 4). We can see that the effect of helium is different in two ranges of $k$. For $k \gtrsim 10^{-4} \mathrm{~m}^{-1}$, thermal conduction is the dominant damping mechanism, so the larger the amount of helium, the smaller $\tau_{\mathrm{D}}$ because of the enhanced thermal conduction by neutral helium atoms. On the other hand, radiative losses are more relevant for $k \lesssim 10^{-4} \mathrm{~m}^{-1}$. In this region, the thermal mode damping time grows as the helium abundance increases. Since these variations in the damping time are very small, we again have to conclude that the damping time obtained in the absence of helium does not significantly change when helium is taken into account. Computations with other values of $\tilde{\mu}_{\mathrm{H}}$ and $\delta_{\mathrm{He}}$ do not modify this statement. We also checked that, as for the slow

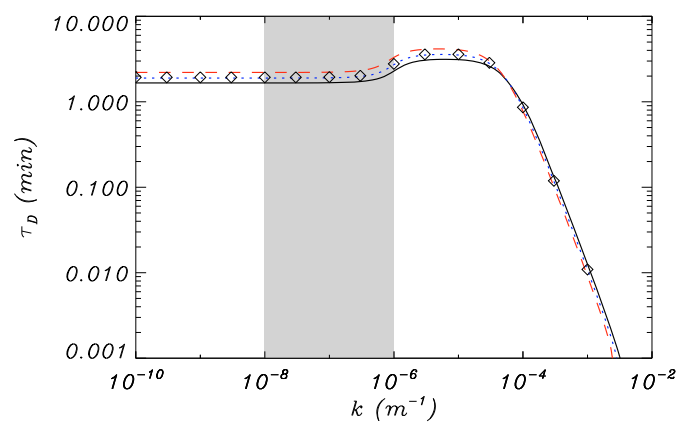

Fig. 4. Damping time, $\tau_{\mathrm{D}}$, of the thermal wave versus the wavenumber, $k$, with $\theta=\pi / 4$. The different linestyles represent: $\xi_{\text {HeI }}=0 \%$ (solid line), $\xi_{\mathrm{He} \text { I }}=10 \%$ (dotted line), and $\xi_{\mathrm{HeI}}=20 \%$ (dashed line). In all computations, $\tilde{\mu}_{\mathrm{H}}=0.8$ and $\delta_{\mathrm{He}}=0.1$. The result for $\xi_{\mathrm{HeI}}=10 \%$ and $\delta_{\mathrm{He}}=0.5$ is plotted by means of symbols for comparison.

mode, the value of the magnetic field strength does not affect the thermal mode damping.

\subsection{Constrained propagation by a waveguide}

We can estimate the effect of a magnetic structure, say a slab or a cylinder, which would act as a waveguide. To do so, we set the wavenumber component in the perpendicular direction to magnetic field lines to a fixed value, $k_{z} L=\pi / 2$, with $L$ a typical length-scale in the perpendicular direction. Since highresolution observations of filaments (see, e.g., Lin et al. 2007, 2009) show fine structures (threads) with a typical width of $\sim 100 \mathrm{~km}$, we select $L=10^{5} \mathrm{~m}$ as our perpendicular lengthscale. Therefore, the propagation angle $\theta$ now depends on $k_{x}$,

$\theta=\arctan \left(\frac{\pi / 2}{k_{x} L}\right)$ 

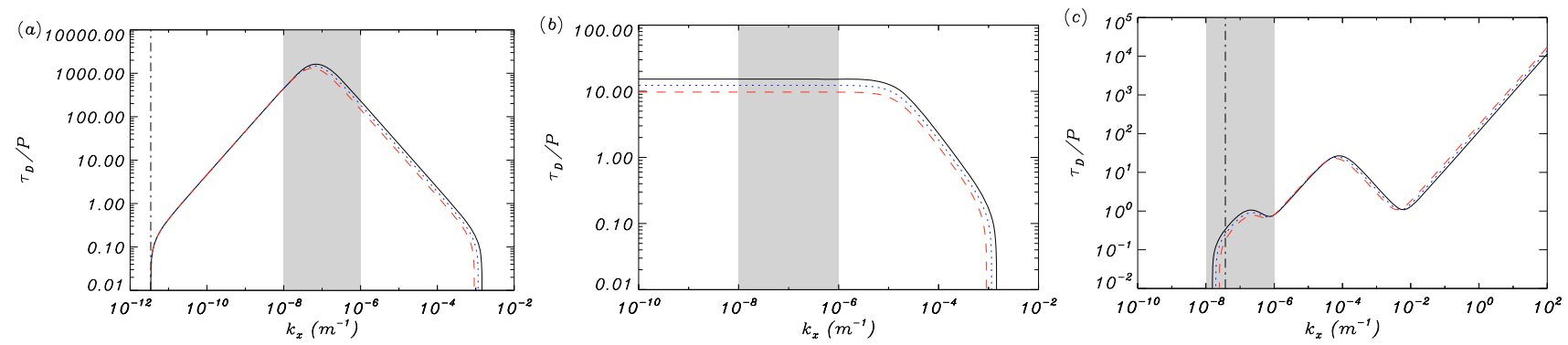

Fig. 5. Ratio of the damping time to the period, $\tau_{\mathrm{D}} / P$, versus the wavenumber component parallel to magnetic field lines, $k_{x}$, corresponding to the a) Alfvén wave, b) fast wave, and c) slow wave for $k_{z} L=\pi / 2$, with $L=10^{5} \mathrm{~m}, \tilde{\mu}_{\mathrm{H}}=0.8$, and $\delta_{\mathrm{He}}=0.1$. The different linestyles represent $\xi_{\mathrm{HeI}}=0$ (solid line), $\xi_{\mathrm{He}}=10 \%$ (dotted line), and $\xi_{\mathrm{HeI}}=20 \%$ (dashed line). The vertical dot-dashed lines in a) and c) correspond to the approximated critical wavenumbers given by Eqs. (29) and (30), respectively, for $\xi_{\mathrm{HeI}}=10 \%$.

Figure 5 displays the results for the Alfvén, fast, and slow waves. We see that the behavior of the three solutions is substantially different from that of the free propagation case. The Alfvén mode now possesses an additional critical wavenumber for low values of $k_{x}$, namely $k_{x}^{\mathrm{c}-}$, which is independent of the ionization degree and the helium abundance. It can be approximated (see details in Soler et al. 2009b, Eq. (38)) as

$k_{x}^{\mathrm{c}-} \approx \frac{\eta}{2 v_{\mathrm{A}}} k_{z}^{2}=\frac{\eta \pi^{2}}{8 v_{\mathrm{A}} L^{2}}$.

On the other hand, the fast wave is now more attenuated in the relevant range of wavenumbers than in the free propagation case, whereas the slow wave also has a new critical wavenumber, namely $k_{x}^{\mathrm{cs}}$, which falls within the relevant range. An expression for the slow mode critical wavenumber is also provided by Eq. (48) of Soler et al. (2009b), which in our present notation is

$k_{x}^{\mathrm{cs}} \approx \frac{c_{\mathrm{s}} \eta_{\mathrm{C}}}{2 v_{\mathrm{A}}^{2}} k_{z}^{2}=\frac{c_{\mathrm{s}} \eta_{\mathrm{C}} \pi^{2}}{8 v_{\mathrm{A}}^{2} L^{2}}$,

where $c_{\mathrm{s}}=\sqrt{\gamma p / \rho}$ is the sound speed, with $\gamma=5 / 3$ the adiabatic index. The slow mode critical wavenumber is shifted toward higher values as the helium abundance increases. There is no additional critical wavenumber for the fast wave. These approximated critical wavenumbers are indicated in Fig. 5. We see excellent agreement in the case of the Alfvén mode critical wavenumber (Eq. (29)). For the slow wave, the approximated value (Eq. (30)) is slightly higher than when numerically obtained. The reason that makes the slow wave critical wavenumber less accurate is found in the simplifications assumed in its derivation (see extensive details in Soler et al. 2009b).

Finally, we also computed the results in the case of the guided thermal disturbance. We find that the thermal mode behavior is the same in the waveguide case and in the free propagation case. As a result, this mode is not affected by the variation of the propagation angle, and no further comments are needed.

\section{Conclusion}

In this work, we have studied the effect of helium (He I and He II) on the time damping of thermal and MHD waves in a partially ionized prominence plasma. This is an extension of previous investigations by Forteza et al. $(2007,2008)$ in which helium was not taken into account. We conclude that, although the neutral helium increases the efficiency of both ion-neutral collisions and thermal conduction, its effect is not important for realistic helium abundances in prominences. In addition, thanks to the very small He II abundance for central prominence temperatures, its presence is irrelevant to the wave behavior. This conclusion applies both to the free propagation case and the constrained propagation by a waveguide case. Although the role of He II (or even He III) could be greater for typical prominence-corona transition region temperatures, the present result allows future studies of MHD waves and oscillations in prominences to neglect the presence of helium.

Acknowledgements. We thank N. Labrosse for giving helpful information about the helium ionization degree in prominences, I. Arregui for some useful comments, and the anonymous referee for constructive suggestions. The authors acknowledge the financial support received from the Spanish MICINN, FEDER funds, and the Conselleria d'Economia, Hisenda i Innovació of the CAIB under Grants No. AYA2006-07637 and PCTIB-2005GC3-03. R.S. thanks the CAIB for a fellowship.

\section{References}

Ballester, J. L. 2006, Phil. Trans. R. Soc. A, 364, 405 Banerjee, D., Erdélyi, R., Oliver R., \& O'Shea, E. 2007, Sol. Phys., 246, 3 Braginskii, S. I. 1965, Rev. Plasma Phys., 1, 205

Carbonell, M., Oliver, R., \& Ballester, J. L. 2004, A\&A, 415, 739

Carbonell, M., Terradas, J., Oliver, R., \& Ballester, J. L. 2006, A\&A, 460, 573

Carbonell, M., Oliver, R., \& Ballester, J. L. 2009, NewA, 14, 277

Cox, D. P., \& Tucker, W. H. 1969, ApJ, 157, 1157

De Pontieu, B., Martens, P. C. H., \& Hudson, H. S. 2001, ApJ, 558, 859

Engvold, O. 2004, Proc. IAU Collq. on Multiwavelength investigations of solar activity, ed. A. V. Stepanov, E. E. Benevolenskaya \& A. G. Kosovichev, 187 Engvold, O. 2008, in Waves \& Oscillations in the Solar Atmosphere: Heating and Magneto-Seismology, ed. R. Erdélyi, \& C. A. Mendoza-Briceño (Cambridge: Cambridge Univ. Press), IAU Symp., 247, 152

Forteza, P., Oliver, R., Ballester, J. L., \& Khodachenko, M. L. 2007, A\&A, 461, 731

Forteza, P., Oliver, R., \& Ballester, J. L. 2008, A\&A, 492, 223

Gouttebroze, P., \& Labrosse, N. 2009, A\&A, 503, 663

Hildner, E. 1974, Sol. Phys., 35, 123

Lin, Y., Engvold, O., Rouppe van der Voort, L. H. M., \& van Noort, M. 2007, Sol. Phys., 246, 65

Lin, Y., Soler, R., Engvold, O., et al. 2009, ApJ, 704, 870

Mackay, D. H., Karpen, J. T., Ballester, J. L., Schmieder, B., \& Aulanier, G. 2010, Space Sci. Rev., accepted [arXiv: 1001.1635]

Oliver, R. 2009, Space Sci. Rev., in press

Oliver, R., \& Ballester, J. L. 2002, Sol. Phys., 206, 45

Parker, E. N. 1953, ApJ, 117, 431

Pinto, C., Galli, D., \& Bacciotti, F. 2008, A\&A, 484, 1

Soler, R., Oliver, R., \& Ballester, J. L. 2007, A\&A, 471, 1023

Soler, R., Oliver, R., \& Ballester, J. L. 2008, ApJ, 684, 725

Soler, R., Oliver, R., \& Ballester, J. L. 2009a, NewA, 14, 238

Soler, R., Oliver, R., \& Ballester, J. L. 2009b, ApJ, 699, 1553

Soler, R., Oliver, R., \& Ballester, J. L. 2009c, ApJ, 707, 662 